\title{
Broadband Spectroscopic Thermoacoustic Characterization of Single-Walled Carbon Nanotubes
}

\author{
Daniel R. Bauer, ${ }^{1,2,3}$ Xiong Wang, ${ }^{4}$ Jeff Vollin, ${ }^{3}$ Hao Xin, ${ }^{4}$ and Russell S. Witte ${ }^{1,2}$ \\ ${ }^{1}$ Department of Medical Imaging, University of Arizona, Tucson, AZ 85724, USA \\ ${ }^{2}$ College of Optical Sciences, University of Arizona, Tucson, AZ 85724, USA \\ ${ }^{3}$ Raytheon Missile Systems, Raytheon Corporation, Tucson, AZ 85736, USA \\ ${ }^{4}$ Department of Electrical and Computer Engineering, University of Arizona, Tucson, AZ 85724, USA
}

Correspondence should be addressed to Daniel R. Bauer; dan.bauer5@gmail.com

Received 6 February 2015; Accepted 27 April 2015

Academic Editor: Nanda Gopal Sahoo

Copyright (C) 2015 Daniel R. Bauer et al. This is an open access article distributed under the Creative Commons Attribution License, which permits unrestricted use, distribution, and reproduction in any medium, provided the original work is properly cited.

\begin{abstract}
Carbon nanotubes have attracted interest as contrast agents for biomedical imaging because they strongly absorb electromagnetic radiation in the optical and microwave regions. This study applies thermoacoustic (TA) imaging and spectroscopy to measure the frequency-dependent absorption profile of single-walled carbon nanotubes (SWNT) in the ranges of $2.7-3.1 \mathrm{GHz}$ and $7-9 \mathrm{GHz}$ using two tunable microwave sources. Between 7 and $9 \mathrm{GHz}$, the peak TA signal for solutions containing semiconducting and metallic SWNTs increased monotonically with a slope of $1.75 \mathrm{AU} / \mathrm{GHz}\left(R^{2}=0.95\right)$ and $2.8 \mathrm{AU} / \mathrm{GHz}\left(R^{2}=0.93\right)$, respectively, relative to a water baseline. However, after compensating for the background signal from water, it was revealed that the TA signal from metallic SWNTs increased exponentially within this frequency band. Results suggest that TA imaging and spectroscopy could be a powerful tool for quantifying the absorption properties of SWNTs and optimizing their performance as contrast agents for imaging or heat sources for thermal therapy.
\end{abstract}

\section{Introduction}

The extraordinary electrical, optical, and mechanical properties of single-walled carbon nanotubes (SWNTs) make them attractive for a variety of applications. For example, they have demonstrated unique capabilities in fields ranging from nanoelectronics for creating highly functional integrated circuits to biomedicine as sensors of in vivo disease biomarkers $[1,2]$. Their low toxicity, large surface area, and ability to penetrate the cell membrane indicate that functionalized SWNTs can be effective agents to deliver exogenous biomolecules like proteins, DNA, and RNA directly into cells [3-7]. These nanoagents can be molecularly conjugated to specific ligands, such as folic acid, antibodies, and peptides, enabling targeted delivery of anticancer or antibacterial agents [8-13]. Additionally, SWNTs have been used in mouse models to deliver therapeutics passively via the enhanced permeability and retention effect (EPR) [14]. In the biomedical arena, these nanoparticles have also been employed as contrast agents for medical imaging and heat sources for thermal ablation due to their strong electromagnetic absorption $[1,15,16]$.
Their large microwave absorption makes SWNTs excellent candidates in contrast to agents for thermoacoustic imaging (TAI) [17-19]. In TAI a short microwave pulse $(\sim 1 \mu \mathrm{s})$ is absorbed by a sample, producing transient heating, thermoelastic expansion, and generation of an ultrasound (US) pressure field with a magnitude proportional to the local microwave absorption. In recent work, we demonstrated that thermoacoustic spectroscopy (TAS) can be used to enhance the specificity of TAI by detecting and identifying materials based on the frequency dependence of their microwave absorption [20]. This study expands our previous findings by utilizing TAS to characterize the spectral dependence of the TA signal generated by SWNTs. The generated TA pressure $(p)$ is given by

$$
p(r, v)=\frac{\beta c_{s}^{2} I(r) \alpha(r, v)}{2 C_{p}}
$$

where $c_{s}$ is the speed of sound, $\beta$ is the isobaric volume expansion coefficient, $C_{p}$ is the specific heat, $I$ is the intensity 
of the microwave pulse, $r$ is the position from the transducer, $\nu$ is the microwave excitation frequency, and $\alpha$ is the microwave absorption coefficient, which is represented by the cumulative contribution from all absorbers:

$$
\alpha(r, v)=\sum_{m=1}^{M} c_{m}(r) \alpha_{m}(r, v),
$$

where $c_{m}$ is the concentration of the $m$ th absorber and $\alpha_{m}$ is the absorption coefficient of the $m$ th absorber. Equation (2) is useful for separating contributions from different colocalized absorbers to the cumulative microwave absorption. The microwave absorption coefficient is defined as

$$
\alpha(v, \mu, \varepsilon, \sigma)=2 \pi v \sqrt{\frac{\mu \varepsilon}{2}\left(\sqrt{1+\left(\frac{\sigma}{2 \pi v \varepsilon}\right)^{2}}-1\right)}
$$

where $\mu$ is the permeability, $\varepsilon$ is the permittivity, and $\sigma$ is the conductivity. Thus, the microwave absorption coefficient and TA signal produced by an absorber are ultimately derived from the underlying dielectric properties of the sample.

Single-walled carbon nanotubes are constructed out of graphene, a two-dimensional sheet of individual carbon atoms arranged in a hexagonal pattern and conceptualized to be rolled into a tube. These nanoparticles typically have a diameter of about one nanometer and can be up to several millimeters in length. Their electromagnetic properties depend on the geometrical orientation of the carbon atoms in the nanotube [21]. For example, carbon nanotubes with a zigzag atomic structure behave as a semiconductor, while carbon nanotubes with an armchair atomic structure behave like a metal.

The spectral absorption properties of SWNTs are largely unexplored. However, previous studies have revealed that their electromagnetic properties, such as permittivity and permeability, can have large frequency dependence in the 1$10 \mathrm{GHz}$ range $[22,23]$. Carbon nanotubes exhibit both linear and nonlinear dielectric properties, so their resulting absorption spectra, as measured by TAS, may be complex in nature [24]. Furthermore, by manipulating the permittivity, permeability, and/or thickness of the nanoparticles, their properties can be frequency-tuned [24-27]. This study employs TAS to ascertain the microwave absorption properties of SWNTs. If these nanoparticles exhibit a distinct spectral signature, this feature could be exploited to enable highly sensitive in vivo discrimination from endogenous background signal generated by tissue (e.g., muscle and blood) [20].

\section{Materials and Methods}

Stock solutions of nanoparticles were obtained from Buckeye Composites containing SWNTs exhibiting semiconducting (MC1138-1) and metallic (MC1138-2) properties. These samples were tested for their frequency-dependent absorption properties using TAS. The nanotubes in the semiconducting solution had an average diameter of $1 \mathrm{~nm}$ and a base concentration of $300 \mathrm{mg} / \mathrm{L}$ water (i.e., $300 \mathrm{ppm}$ ). The nanotubes in the metallic solution had an average diameter of $2 \mathrm{~nm}$ and a concentration of $100 \mathrm{mg} / \mathrm{L}$ water (i.e., $100 \mathrm{ppm}$ ).

Two microwave sources were employed for TAI with excitation frequencies between 2.7 and $9 \mathrm{GHz}$. The first source was a high-energy microwave oscillator (PG5KB, Epsco, $P_{\text {peak }}=4 \mathrm{~kW}$, Figure 1(a)) tunable between 2.7 and $3.1 \mathrm{GHz}$. The second source was a high-frequency microwave power amplifier provided by Raytheon Company (see Figure 1(b)). This source exploited a helix traveling wave tube (TWT) amplifier to generate microwave pulses with a peak power of $1 \mathrm{~kW}$ between 7 and $9 \mathrm{GHz}$.

For TAI, a custom tank was designed and fabricated on a rapid prototype $3 \mathrm{D}$ printer (Connex350, Objet). A singleelement, spherically focused 2.25 MHz US transducer (V395, Olympus) detected the TA signals, which were generated with a $300 \mathrm{~ns}$ square microwave excitation pulse. The radiating antenna, a WR-187 waveguide for the $3 \mathrm{GHz}$ system, was filled with mineral oil and sealed with a thin layer of Mylar. A ceramic matching layer (Eccostock $\mathrm{HiK}$, Emerson \& Cuming, $\varepsilon_{r}=6.0$, loss tangent $<0.002$ ) was positioned on top of the waveguide to optimize microwave transmission into the sample. A calibrated spectrum analyzer (Hewlett-Packard, $8560 \mathrm{~A}$ ) measured the frequency-dependent power of the microwave source. The imaging tank was filled with deionized water $\left(\mathrm{diH}_{2} \mathrm{O}\right)$ for acoustic coupling to the US transducer. The setup for the $3 \mathrm{GHz}$ system, including imaging tank, overhead transducer, and arm of the motorized 3D translation stage (Velmex Inc.), is displayed in Figure 1(c).

For the $9 \mathrm{GHz}$ system, the imaging tank was fitted with a WR-90 waveguide. Both the waveguide and imaging tank were filled with mineral oil and separated by a thin layer of Mylar. The microwave output was sampled with a directional coupler. The attenuated output of the coupler was connected to a crystal detector (432A, Hewlett Packard) to convert the microwave power to a voltage. The crystal detector was calibrated at each microwave frequency and used to record the frequency-dependent fluctuations in the output microwave power. It also enabled accurate tuning of the microwave pulse width.

For both systems, US signals were recorded on a fast data acquisition board (PDA12, Signatec), which sampled signals at $32 \mathrm{MHz}$. During TAI, the envelope of the output microwave pulse was also recorded. The lateral position of the transducer was placed at the center of the waveguide where the microwave power was highest. The acoustic focus was then positioned at the depth of the sample. The motorized translation stage was attached to the sample, which was scanned across the waveguide aperture to ensure uniform and consistent microwave exposure for each sample. Next, the sample was scanned at different microwave excitation frequencies to produce spectroscopic cross-sectional (B-mode) TA images. The sample consisted of three polyolefin tubes (NTE Electronics, dia. $=3 \mathrm{~mm}$ ) with solutions of semiconducting SWNTs, metallic SWNTs, and deionized water (see Figure 1(c)). The sample was suspended $2 \mathrm{~mm}$ above the waveguide aperture with a center-to-center spacing of $5 \mathrm{~mm}$ between each tube. The typical scan time for a single Bmode acquisition was less than 1 minute with lateral steps of $500 \mu \mathrm{m}$. The US signal at each lateral position was bandpass 


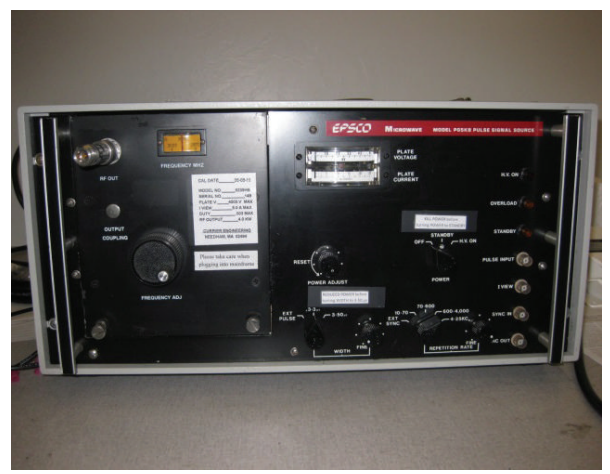

(a)

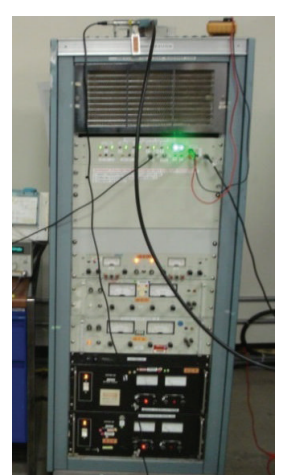

(b)

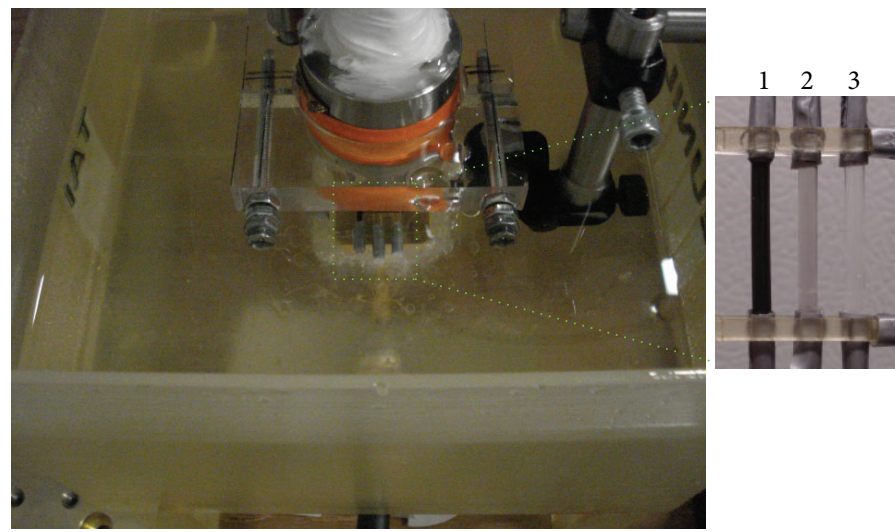

(c)

FIgURE 1: (a) Photograph of the 2.7-3.1 GHz tunable microwave source. (b) Photograph of the 7-9 GHz tunable microwave source (traveling wave tube oscillator). The amplified microwave output was connected to a waveguide (WR-90 for 7-9 GHz), which was secured to the bottom of the imaging tank. (c) Photograph of imaging tank and single-element focused US transducer. Highlighted region: photograph of sample consisting of solutions of (1) semiconducting SWNTs, (2) metallic SWNTs, and (3) a baseline of deionized water. All solutions were imaged in $3 \mathrm{~mm}$ diameter polyolefin tubes. The solution of semiconducting SWNTs is darker than the solution of metallic SWNTs partly due to the higher nanoparticle concentration (300 ppm versus $100 \mathrm{ppm})$.

filtered (1-3 MHz) and demodulated by taking the magnitude of the Hilbert transform. For display purposes, the envelope was linearly interpolated and log-compressed. Images were filtered laterally with a low-order median filter followed by a Gaussian smoothing filter $(1.5 \mathrm{~mm})$. Thermoacoustic images were normalized to the power of the microwave pulse, which varied slightly with frequency.

\section{Results and Discussion}

Solutions of SWNTs were initially imaged with the tunable $3 \mathrm{GHz}$ microwave source. A test sample consisting of solutions of semiconducting SWNTs and metallic SWNTs and a reference solution of deionized water was imaged with excitation frequencies of 2.7, 2.9, and $3.1 \mathrm{GHz}$. A schematic cross-section of the samples is shown in Figure 2(a), displaying the order of each solution. A TA image acquired with $3.1 \mathrm{GHz}$ radiation is presented in Figure 2(b). The tube of semiconducting SWNTs generated the largest TA signal of the three samples. This is consistent with expectations, because its nanoparticle concentration was higher than that of the metallic nanoparticles. For example, at $3.1 \mathrm{GHz}$, this sample generated $48 \%$ and $90 \%$ larger signal than the metallic and water samples, respectively. Additionally, the normalized spectral profiles of the SWNTs between 2.7 and $3.1 \mathrm{GHz}$ are depicted in Figure 2(c). Both SWNT solutions generated significantly larger signal $(20 \%-100 \%)$ than the water reference. Over this narrow frequency range, the spectrum of each sample increased linearly $\left(R^{2} \geq 0.99\right)$ but with different frequency-dependent slopes. Referenced to the unit normalized slope of deionized water, the metallic SWNT solution had a spectral slope of $0.95 \mathrm{AU} / \mathrm{GHz}$, which was slightly less than that of water, whereas the semiconducting SWNT solution had a slope significantly greater than the water baseline (2.32 AU/GHz).

To further explore the spectral absorption properties of the SWNTs, the nanoparticle solutions were imaged with the broadband TWT oscillator. Thermoacoustic images, displayed in Figure 3, were acquired with excitation frequencies between 7 and $9 \mathrm{GHz}$ in $0.25 \mathrm{GHz}$ intervals. These images indicate that the solutions of SWNTs generated stronger TA signals than deionized water, especially above $8 \mathrm{GHz}$. This is particularly significant because water itself has substantial absorption in this frequency range. 


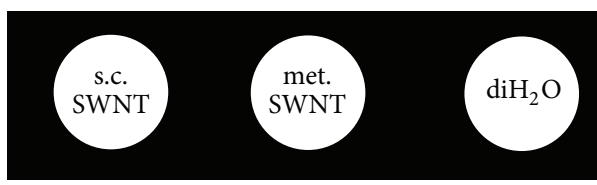

(a)

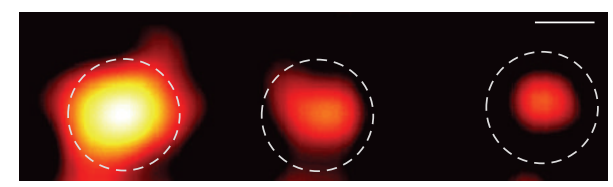

(b)

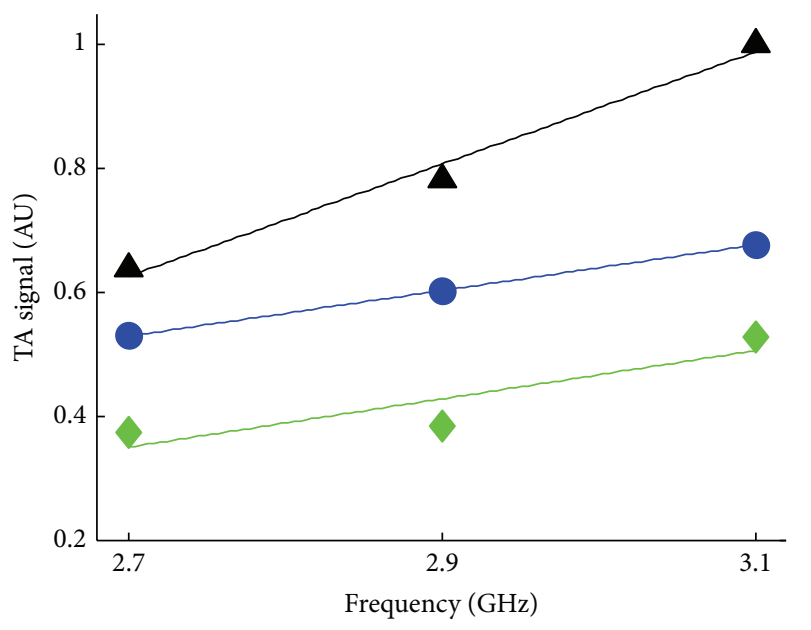

s.c. SWNT
met. SWNT
$\mathrm{diH}_{2} \mathrm{O}$

(c)

FIGURE 2: (a) Schematic cross-section of sample imaged in Figure 1(c) comprised of semiconducting SWNTs (s.c. SWNT, 300 ppm), metallic SWNTs (met. SWNT, $100 \mathrm{ppm}$ ), and a baseline control of deionized water $\left(\mathrm{diH}_{2} \mathrm{O}\right)$. (b) TA image acquired with $3.1 \mathrm{GHz}$ microwave radiation ( $8 \mathrm{~dB}$ dynamic range). Tubes are outlined by gray circles. Scale bar $=2 \mathrm{~mm}$. (c) Average TA signal, over $4 \times 4 \mathrm{~mm}^{2}$ regions for semiconducting SWNTs $\left(m=2.32 \mathrm{AU} / \mathrm{GHz}, R^{2}=0.99\right)$ and metallic SWNTs $\left(m=0.95 \mathrm{AU} / \mathrm{GHz}, R^{2}=0.99\right)$ relative to the normalized slope of deionized water $\left(m=1.0 \mathrm{AU} / \mathrm{GHz}, R^{2}=0.80\right)$ as a function of incident microwave frequency.

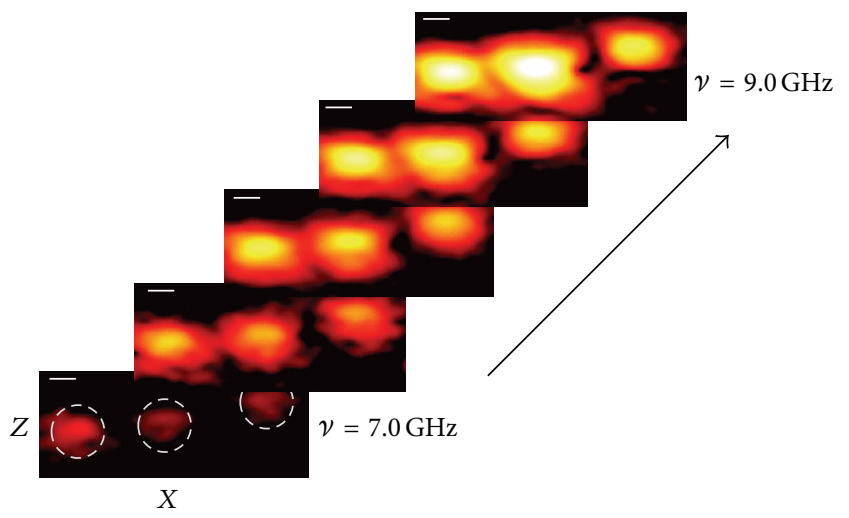

Figure 3: Spectroscopic TA cross-sectional images of semiconducting SWNTs (left tube), metallic SWNTs (middle tube), and deionized water (right tube), acquired with excitation frequencies between 7 and $9 \mathrm{GHz}(\Delta \nu=0.5 \mathrm{GHz})$. Tube walls are outlined by gray circles in bottom image. A strong spectral dependence is observed for each sample. Dynamic range is $30 \mathrm{~dB}$ for all, scale bar $=2 \mathrm{~mm}$.

The TA signal's amplitude at each microwave frequency, averaged over the cross-section of each tube, is displayed in Figure 4(a). Both solutions of SWNTs manifested a TA signal that increased linearly as a function of microwave frequency.
The stock solutions of semiconducting and metallic SWNTs had respective slopes of $1.75 \mathrm{AU} / \mathrm{GHz}\left(R^{2}=0.95, p<0.01\right)$ and $2.8 \mathrm{AU} / \mathrm{GHz}\left(R^{2}=0.93, p<0.01\right)$ compared to the unit normalized slope of deionized water. Figure 4(b) presents an image of the sample cross-section based on the slope of the best-fit line at each pixel. The three samples have distinct spectral signatures, allowing each to be uniquely identified from their frequency-dependent TA signal without relying on the magnitude of the signal at any particular frequency.

Because most of the solutions were composed of water (>99\% by weight) with significant background absorption, we also estimated the individual contribution of the nanoparticles to the cumulative TA signal. Equation (2) was used to decompose the contributions of the total TA signal into two discrete sources of microwave absorption (i.e., water and SWNTs) using the known nanoparticle concentration of each solution $\left(c_{\text {s.c. } \text { SWNT }}=300 \mathrm{ppm}, c_{\text {met. SWNT }}=100 \mathrm{ppm}\right)$ and the measured TA spectrum of deionized water. This approach also compensates for different nanoparticle concentrations and, thereby, enables better quantification of the nanoparticle's true absorption profile. The expected TA signals from samples of pure SWNTs are displayed in Figure 5 on the same scale as Figure 4. The semiconducting SWNTs exhibited a strong absorption profile that increased linearly with frequency. The metallic SWNTs, on the other hand, revealed 

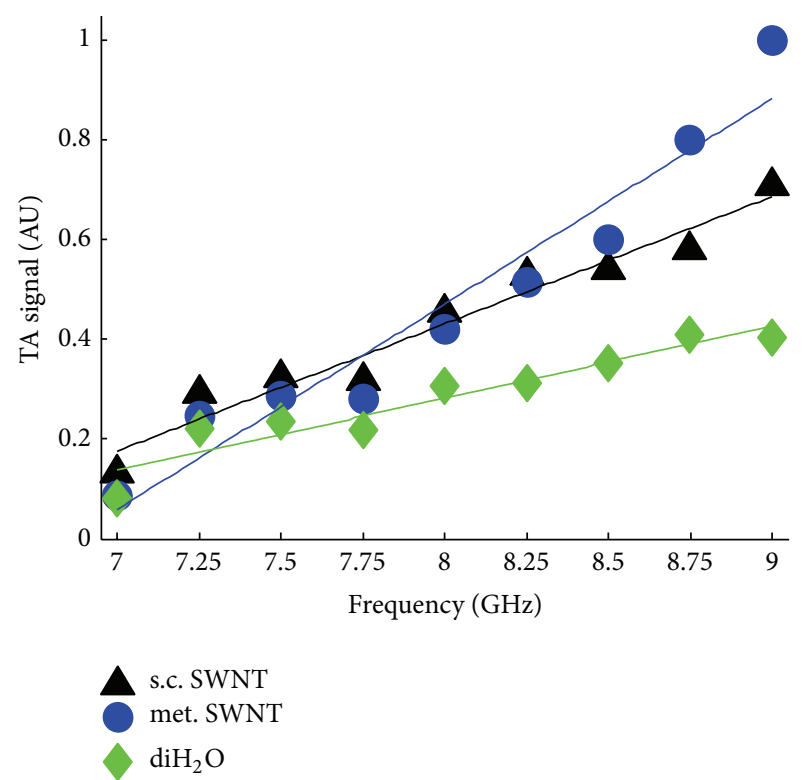

(a)

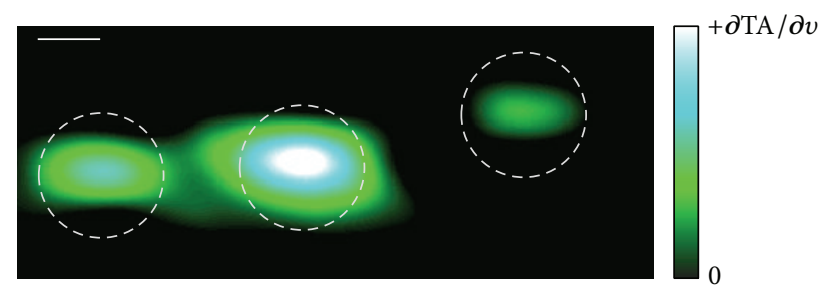

(b)

Figure 4: (a) Average TA signal from semiconducting SWNTs, metallic SWNTs, and deionized water. The semiconducting and metallic SWNT solutions had respective slopes of $1.75 \mathrm{AU} / \mathrm{GHz}$ $\left(R^{2}=0.95\right)$ and $2.8 \mathrm{AU} / \mathrm{GHz}\left(R^{2}=0.93\right)$, with respect to the water baseline (i.e., $m_{\text {water }}=1.0 \mathrm{AU} / \mathrm{GHz}, R_{\mathrm{diH}_{2} \mathrm{O}}^{2}=0.91$ ); $p<0.01$ for all three samples. (b) Cross-sectional image of the slope of the TA signal, as a function of microwave excitation frequency, as calculated from the pixel-wise least-squares best-fit line. Tube cross-sections are outlined by dashed gray circles.

a much stronger signal than both deionized water and semiconducting SWNTs between 7 and $9 \mathrm{GHz}$ with the TA signal increasing exponentially within this frequency range.

Although the TA signal is directly proportional to the absorption coefficient of each sample, it is not a direct measure of microwave absorption due to other factors that affect the TA signal, such as the thermoelastic expansion and specific heat (see (1)). However, we expected the frequencydependence of the microwave absorption between 1 and $10 \mathrm{GHz}$ to be dominated by changes in its absorption properties. The linear absorption spectrum of semiconducting nanoparticles near $3 \mathrm{GHz}$ and between 7 and $9 \mathrm{GHz}$ is consistent with previous reports describing slowly varying dielectric properties for SWNTs in this frequency range [24]. Conversely, TAI and spectroscopy revealed an exponentially increasing signal for the metallic SWNTs between 7 and $9 \mathrm{GHz}$. Other studies have reported spectrally varying dielectric properties of SWNTs near this frequency range using

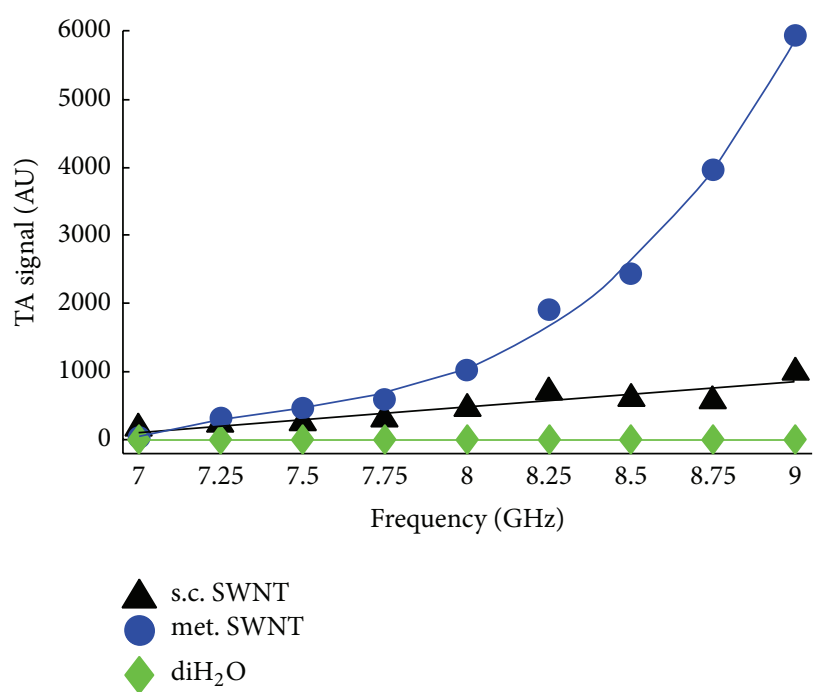

Figure 5: Expected TA spectra of SWNTs calculated by compensating for contributions from the background water in each SWNT solution. The water and semiconducting SWNTs have a linear spectra $\left(m_{\text {water }}=0.145 \mathrm{AU} / \mathrm{GHz}, R^{2}=0.91\right)$ and $\left(m_{\text {s.c. } \text { SWNT }}=\right.$ $366 \mathrm{AU} / \mathrm{GHz}, R^{2}=0.84$ ), respectively. The frequency-dependent signal from metallic SWNTs followed an exponential function (halflife $=0.49 \mathrm{GHz}, R_{\text {adj }}^{2}=0.84$ ).

other methods, such as field emission scanning electron microscopy $[24,25,27,28]$. The exact mechanism of the increase could be explained by a plasmon resonance, specific to the size and distribution of the metallic SWNTs, modulating the attenuation coefficient of the nanoparticles [29]. The sharp spectral signature and large signal enhancement of metallic SWNTs may facilitate clinical application of contrast-enhanced TAS for diagnostic cancer imaging. Exploiting their frequency-dependent absorption may also improve their heating efficiency for deeper imaging and therapy in soft tissue using high-frequency microwaves.

\section{Conclusions}

This study employed spectroscopic thermoacoustic imaging to characterize the spectral dependence of semiconducting and metallic single-walled carbon nanotubes (SWNTs) between 2.7 and $9 \mathrm{GHz}$. These nanoparticles were found to have distinct and differentiable absorption spectra in this frequency range. In particular, after compensating for effects of the background water signal, metallic SWNTs displayed an exponentially increasing thermoacoustic signal between 7 and $9 \mathrm{GHz}$. In tissue, the strong and distinct microwave absorption properties of metallic SWNTs could be separated from endogenous background absorbers (e.g., blood, muscle, and fat) using spectral decomposition to facilitate in vivo detection of small tumors. Molecularly targeted SWNTs combined with thermoacoustic spectroscopy could lead to highly sensitive and specific detection and imaging of cancer cells followed by delivery of therapeutics. These agents could also be used with microwave heating for targeted ablation and cancer therapy. 


\section{Conflict of Interests}

The authors declare that there is no conflict of interests regarding the publication of this paper.

\section{Acknowledgments}

The authors are grateful to Raytheon Corporation for providing access to their $7+\mathrm{GHz}$ microwave transmitter, which made this study possible. They also acknowledge the Center for Gamma Ray Imaging for maintaining and running the rapid prototyping printer, which was used to fabricate the imaging tank for this study.

\section{References}

[1] Z. Liu, S. Tabakman, K. Welsher, and H. Dai, "Carbon nanotubes in biology and medicine: in vitro and in vivo detection, imaging and drug delivery," Nano Research, vol. 2, no. 2, pp. 85$120,2009$.

[2] H. Gong, R. Peng, and Z. Liu, "Carbon nanotubes for biomedical imaging: the recent advances," Advanced Drug Delivery Reviews, vol. 65, no. 15, pp. 1951-1963, 2013.

[3] M. L. Schipper, N. Nakayama-Ratchford, C. R. Davis et al., "A pilot toxicology study of single-walled carbon nanotubes in a small sample of mice," Nature Nanotechnology, vol. 3, no. 4, pp. 216-221, 2008.

[4] N. W. S. Kam, T. C. Jessop, P. A. Wender, and H. Dai, "Nanotube molecular transporters: Internalization of carbon nanotube-protein conjugates into mammalian cells," Journal of the American Chemical Society, vol. 126, no. 22, pp. 6850-6851, 2004.

[5] H. Jin, D. A. Heller, and M. S. Strano, "Single-particle tracking of endocytosis and exocytosis of single-walled carbon nanotubes in NIH-3T3 cells," Nano Letters, vol. 8, no. 6, pp. 1577-1585, 2008.

[6] M. K. Gottipati, E. Bekyarova, R. C. Haddon, and V. Parpura, "Chemically functionalized single-walled carbon nanotubes enhance the glutamate uptake characteristics of mouse cortical astrocytes," Amino Acids, 2015.

[7] D. Scheinberg, J. Mulvey, E. Feinberg, S. Alidori, D. Heller, and M. McDevitt, "Synthesis, pharmacokinetics, and biological use of lysine-modified single-walled carbon nanotubes," International Journal of Nanomedicine, vol. 9, no. 1, pp. 4245-4255, 2014.

[8] S. Dhar, Z. Liu, J. Thomale, H. Dai, and S. J. Lippard, "Targeted single-wall carbon nanotube-mediated $\mathrm{Pt}(\mathrm{IV})$ prodrug delivery using folate as a homing device," Journal of the American Chemical Society, vol. 130, no. 34, pp. 11467-11476, 2008.

[9] M. R. McDevitt, D. Chattopadhyay, B. J. Kappel et al., “Tumor targeting with antibody-functionalized, radiolabeled carbon nanotubes," The Journal of Nuclear Medicine, vol. 48, no. 7, pp. 1180-1189, 2007.

[10] Z. Liu, W. Cai, L. He et al., "In vivo biodistribution and highly efficient tumour targeting of carbon nanotubes in mice," Nature Nanotechnology, vol. 2, no. 1, pp. 47-52, 2007.

[11] Z. Liu, K. Chen, C. Davis et al., "Drug delivery with carbon nanotubes for in vivo cancer treatment," Cancer Research, vol. 68, no. 16, pp. 6652-6660, 2008.

[12] Z. H. Zhang, X. Y. Yang, Y. Zhang et al., "Delivery of telomerase reverse transcriptase small interfering RNA in complex with positively charged single-walled carbon nanotubes suppresses tumor growth," Clinical Cancer Research, vol. 12, no. 16, pp. 4933-4939, 2006.

[13] W. Wu, S. Wieckowski, G. Pastorin et al., "Targeted delivery of amphotericin B to cells by using functionalized carbon nanotubes," Angewandte Chemie-International Edition, vol. 44, no. 39, pp. 6358-6362, 2005.

[14] B. R. Smith, E. E. B. Ghosn, H. Rallapalli et al., "Selective uptake of single-walled carbon nanotubes by circulating monocytes for enhanced tumour delivery," Nature Nanotechnology, vol. 9, pp. 481-487, 2014.

[15] P. Chakravarty, R. Marches, N. S. Zimmerman et al., “Thermal ablation of tumor cells with antibody-functionalized singlewalled carbon nanotubes," Proceedings of the National Academy of Sciences of the United States of America, vol. 105, no. 25, pp. 8697-8702, 2008.

[16] C. Liang, S. Diao, C. Wang et al., "Tumor metastasis inhibition by imaging-guided photothermal therapy with single-walled carbon nanotubes," Advanced Materials, vol. 26, no. 32, pp. 5646-5652, 2014.

[17] M. Pramanik, M. Swierczewska, D. Green, B. Sitharaman, and L. V. Wang, "Single-walled carbon nanotubes as a multimodalthermoacoustic and photoacoustic-contrast agent," Journal of Biomedical Optics, vol. 14, no. 3, pp. 1-8, 2009.

[18] O. Ogunlade and P. Beard, "Exogenous contrast agents for thermoacoustic imaging: an investigation into the underlying sources of contrast," Medical Physics, vol. 42, no. 1, pp. 170-180, 2015.

[19] J. Song, Z. Zhao, J. Wang et al., "Evaluation of contrast enhancement by carbon nanotubes for microwave-induced thermoacoustic tomography," IEEE Transactions on Biomedical Engineering, vol. 62, no. 3, pp. 930-938, 2015.

[20] D. R. Bauer, X. Wang, J. Vollin, H. Xin, and R. S. Witte, "Spectroscopic thermoacoustic imaging of water and fat composition," Applied Physics Letters, vol. 101, no. 3, Article ID 033705, 4 pages, 2012.

[21] A. K.-T. Lau and D. Hui, "The revolutionary creation of new advanced materials - carbon nanotube composites," Composites Part B:Engineering, vol. 33, no. 4, pp. 263-277, 2002.

[22] A. Mashal, B. Sitharaman, J. H. Booske, and S. C. Hagness, "Dielectric characterization of carbon nanotube contrast agents for microwave breast cancer detection," in Proceedings of the IEEE Antennas and Propagation Society International Symposium (APSURSI '09), pp. 1-4, IEEE, Charleston, SC, USA, June 2009.

[23] K. He, L. Yu, L. Sheng, K. An, Y. Ando, and X. Zhao, "Doping effect of single-wall carbon nanotubes on the microwave absorption properties of nanocrystalline barium ferrite," Japanese Journal of Applied Physics, vol. 49, no. 12R, Article ID 125101, 2010.

[24] P. Theilmann, K. M. Chu, P. R. Bandaru, P. Asbeck, and S. H. Park, "Optimisation of microwave absorption of carbon nanotube composites through use of carboxyl-epoxide functional group linkages," Electronics Letters, vol. 48, no. 11, pp. 638-639, 2012.

[25] Y. Rui-Gang, "Superparamagnetic nanoparticles for biomedical applications: possibilities and limitations of a new drug delivery system," Journal of Magnetism and Magnetic Materials, vol. 293, pp. 483-496, 2005.

[26] A. Saib, L. Bednarz, R. Daussin et al., "Carbon nanotube composites for broadband microwave absorbing materials," IEEE 
Transactions on Microwave Theory and Techniques, vol. 54, no. 6, pp. 2745-2754, 2006.

[27] H. Zhang, G. Zeng, Y. Ge, T. Chen, and L. Hu, "Electromagnetic characteristic and microwave absorption properties of carbon nanotubes/epoxy composites in the frequency range from 2 to $6 \mathrm{GHz}$," Journal of Applied Physics, vol. 105, no. 5, Article ID 054314, 2009.

[28] P. Bhattacharya, S. Sahoo, and C. K. Das, "Microwave absorption behaviour of MWCNT based nanocomposites in X-band region," Express Polymer Letters, vol. 7, no. 3, pp. 212-223, 2013.

[29] P. J. Burke, "Lüttinger liquid theory as a model of the gigahertz electrical properties of carbon nanotubes," IEEE Transactions on Nanotechnology, vol. 1, no. 3, pp. 129-144, 2002. 

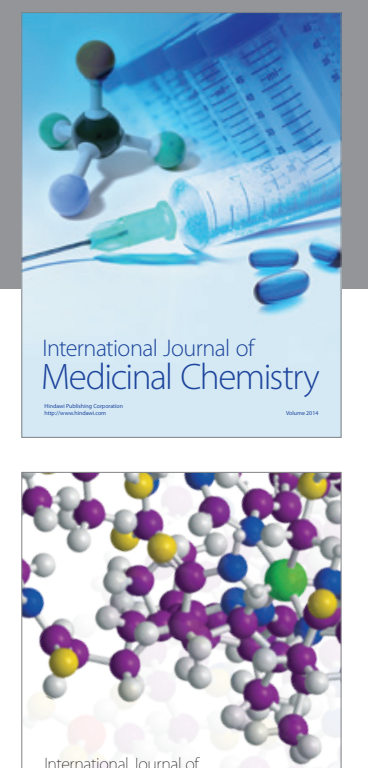

\section{Carbohydrate} Chemistry

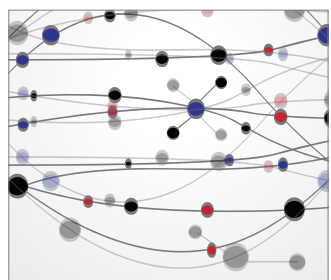

The Scientific World Journal
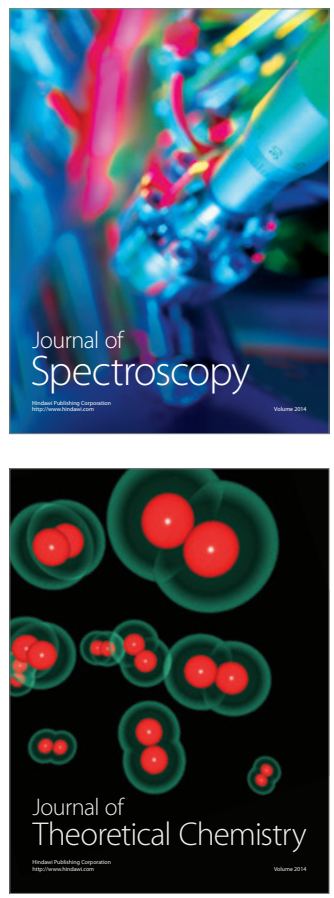
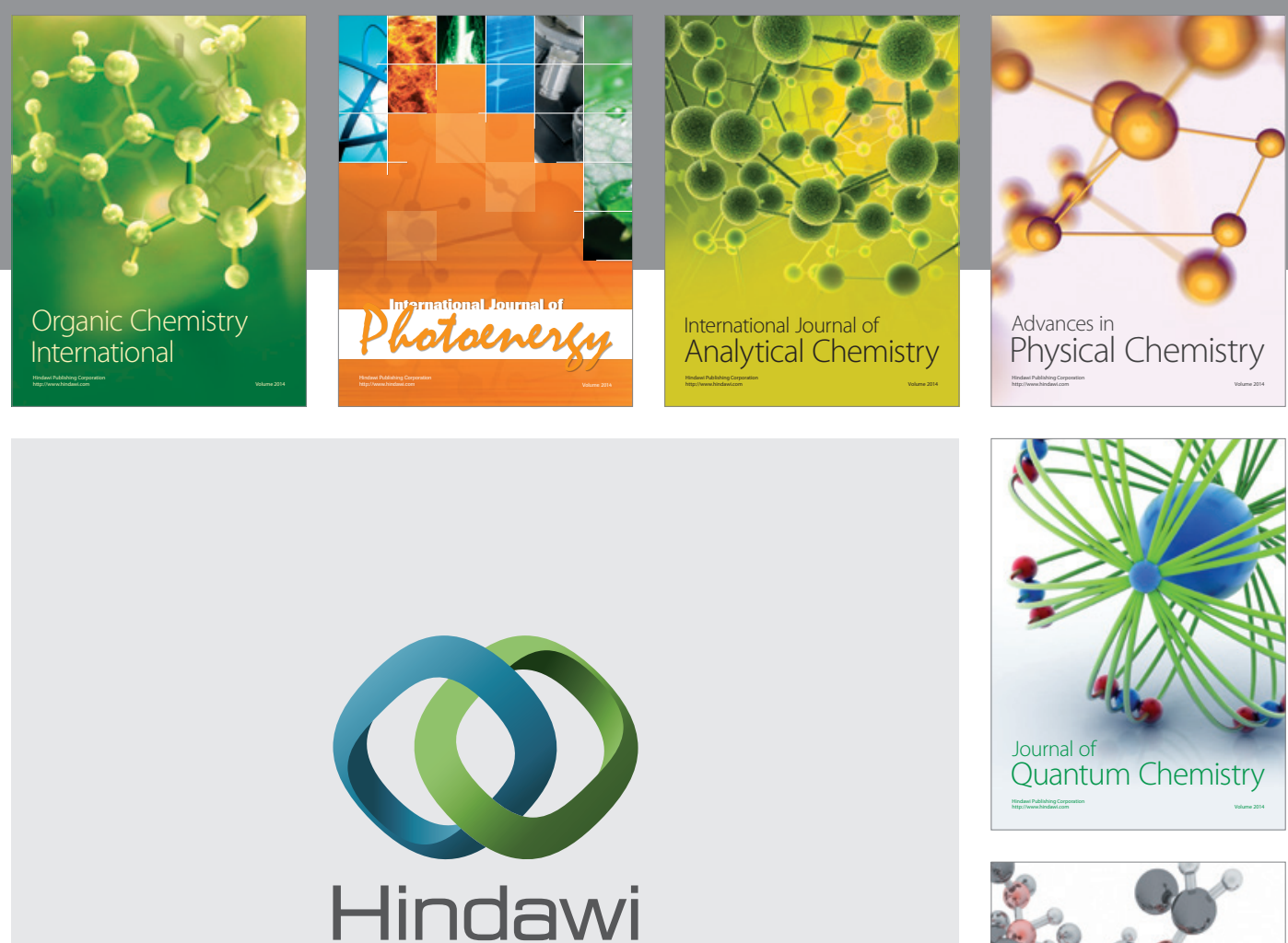

Submit your manuscripts at

http://www.hindawi.com

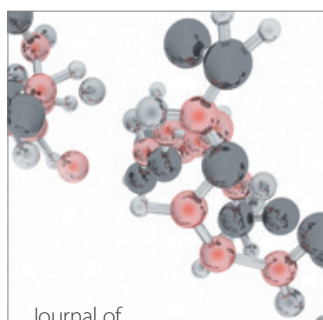

Analytical Methods

in Chemistry

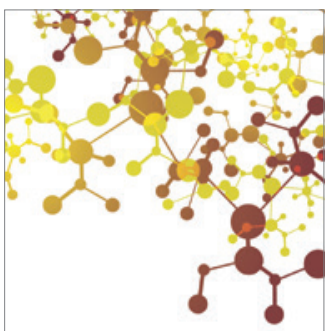

Journal of

Applied Chemistry

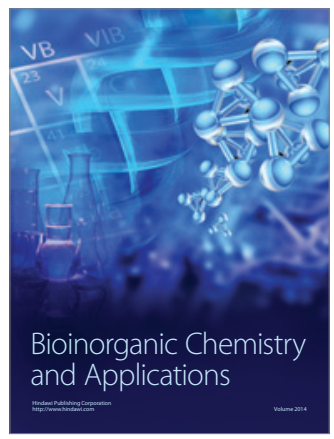

Inorganic Chemistry
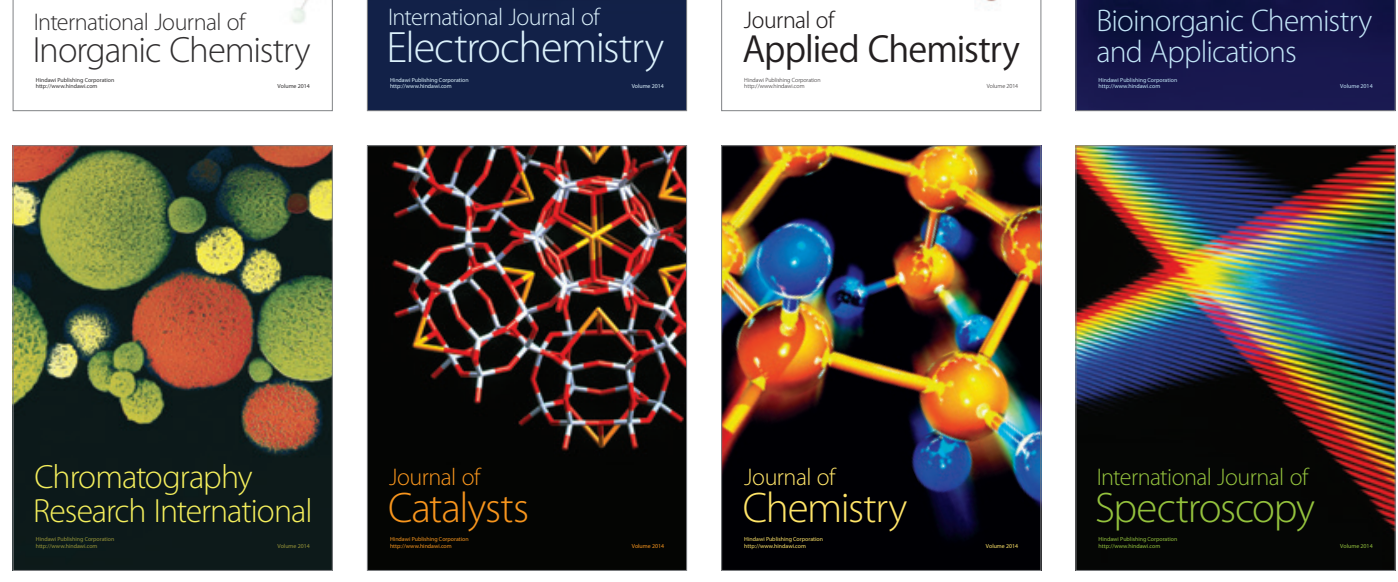